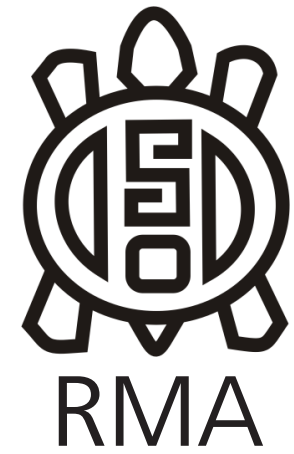

Arqueología

\title{
Análisis de cueros arqueológicos en sitios de frontera de la pampa seca (Argentina, fines de siglo XIX)
}

\author{
Analysis of archaeological leather in pampa seca frontier sites \\ (Argentina, end of XIX century) \\ Jimena Doval ${ }^{*}$ y Alicia Tapia ${ }^{* *}$
}

*Instituto de Arqueología, Facultad de Filosofía y Letras, Universidad de Buenos Aires.

E-mail: dovaljimena@gmail.com

"Instituto de Arqueología, Facultad de Filosofía y Letras, Universidad de Buenos Aires.

Departamento de Ciencias Sociales, Universidad Nacional de Luján.

E-mail: aliciahtapia@yahoo.com.ar

\begin{abstract}
Resumen
Se presentan los resultados del análisis efectuado a fragmentos de cuero recuperados en los sitios Don Isidoro 2 y Fortín La Perra (La Pampa, Argentina). La muestra de cueros de ambos sitios asciende a 83 fragmentos, aunque algunos de ellos pertenecieron al mismo tipo de objeto (correajes, calzados, etc.). Para el estudio arqueométrico se seleccionaron 32 muestras de cuero. La identificación taxonómica de las muestras se efectuó en el CITEC (INTI) a partir del análisis macroscópico y microscópico de la capa flor y la capa corium. Asimismo, se realizó el análisis tecno-morfológico de los fragmentos tomando en consideración diversas variables. Los resultados obtenidos permiten identificar el taxón y la posible función que habrían cumplido algunos de los artefactos de cuero. A su vez, aportan información sobre las prácticas sociales desarrolladas a fines de siglo XIX por los diversos actores sociales en el espacio fronterizo.
\end{abstract}

Palabras clave: cueros; taxonomía; tafonomía; Arqueología histórica; fuentes documentales.

\begin{abstract}
We present the results of the analysis carried out on recovered leather fragments at Don Isidoro 2 and Fortín La Perra sites (La Pampa, Argentina). The sample of leathers from both sites amounts to 83 fragments and technomorphological and archaeometric analysis were carried out to study them. A total of 32 leather samples were selected and analyzed in the CITEC (INTI) for the archaeometric study, by means of a macroscopic and microscopic analysis of the grain layer and the corium layer, to determine their taxonomy and work processes. The technomorphological analysis of the fragments took into consideration several study variables. The results we obtained enabled us to identify the taxon and the possible function that some of the leather goods would have fulfilled. Finally, we put together all the archaeometric data with those provided by the documentary sources; so, we could draw a conclusion about some social practices developed at the end of the 19th century by the various social actors in the frontier space.
\end{abstract}

Key words: leather; taxonomy; taphonomy; historical archaeology; documentary sources.

Desde momentos tempranos el cuero se ha configurado como uno de los recursos de origen animal utilizado por el hombre como materia prima para la confección de vestimenta, viviendas, transporte y otros artefactos para diversos usos. No obstante, como todo material orgánico su hallazgo y conservación están sujetos a las condiciones de preservación de los diversos contextos arqueológicos. En la arqueología argentina, los estudios zooarqueológicos se han centrado casi exclusivamente en el análisis de los restos óseos, siendo escasos los trabajos sobre fibras y cueros faunísticos. Al respecto, en este estudio se presentan los resultados del análisis de fragmentos de cueros provenientes de dos sitios arqueológicos ubicados en el norte de la pampa seca, ambos correspondientes a fines de siglo XIX (Figura 1). A partir del estudio de restos de vestimenta, calzado y otros artefactos confeccionados con cuero se espera dar cuenta de algunas prácticas sociales que tuvieron lugar en una toldería indígena y en un fortín militar. En este sentido, el registro arqueológico hallado en los diferentes asentamientos de frontera puede proporcionar información sobre la diversidad y complejidad social en el espacio fronterizo, comprender la vida cotidiana, las interrelaciones entre los diferentes actores sociales y 
los cambios producidos a lo largo del tiempo. Para ello se realizará un abordaje multidisciplinario utilizando técnicas arqueométricas destinadas a identificar tanto la taxonomía de las diferentes muestras como las diversas modificaciones post-depositacionales que afectaron a los materiales.

A continuación se presentan algunos antecedentes sobre los casos de estudio que abordan el análisis de cueros y fibras arqueológicas en nuestro país. Luego se indican los contextos arqueológicos de donde provienen las muestras analizadas, se explicitan los procedimientos metodológicos seguidos $y$, finalmente, se detallan y discuten los resultados obtenidos integrando los datos arqueológicos y documentales.

\section{Antecedentes}

El estudio de cueros arqueológicos realizados en nuestro país es escaso y prácticamente inexistente en el marco de la Arqueología histórica. Si bien el hallazgo de cueroscomo de otros materiales orgánicos- depende de las condiciones de conservación de los sitios, en muchos casos, aunque se han recuperado materiales de este tipo no se efectuaron estudios arqueométricos primando una perspectiva descriptiva. A la escasez de estudios de los cueros arqueológicos se suma la falta de profundización sobre los aspectos metodológicos y tipológicos (Fernández Distel 2001). En algunos trabajos se analizaron los cueros de modo indirecto, a partir del análisis funcional o de las trazas residuales sobre los artefactos líticos o de

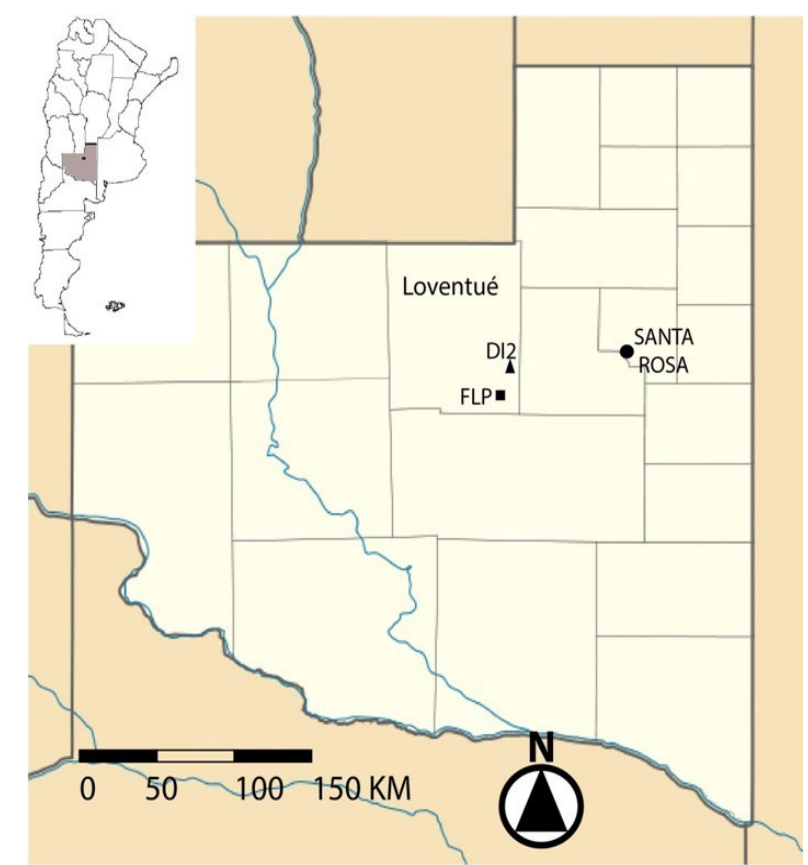

Figura 1. Ubicación de los sitios arqueológicos en los cuales fueron recuperados los cueros que se analizan: Don Isidoro 2 (DI2) y Fortín La Perra (FLP), Departamento Loventué, La Pampa.

Figure 1. Location of the archaeological sites, where the leathers we analyze were recovered: Don Isidoro 2 (DI2) and Fortín La Perra (FLP), Loventué Department, La Pampa. hueso (Buc 2005; Gómez Romero 2007; Cattaneo y Aguerre2009; Hermo y Lynch 2014; entre otros). Una gran cantidad de trabajos se han centrado en el análisis desde la perspectiva etnoarqueológica y a través del análisis de relatos de cronistas y viajeros (Yacobaccio et al. 1998; Aguerre 2000; Casamiquela2000; Caviglia 2002; Prates 2009; entre otros).

Desde una perspectiva descriptiva se encuentran los trabajos de Casamiquela (2000) sobre los toldos de indígenas de Patagonia y Pampa y de Fernández Distel (2001) sobre calzados hallados en asentamientos de la Puna Argentina. Por su parte Caviglia (2002), ha analizado detalladamente las capas pintadas Tehuelches y la importancia significativa de las pinturas decorativas en la reproducción de su cosmovisión. El trabajo de Ciampagna et al. (2006) presenta la descripción del primer y único hallazgo de un cuero arqueológico para un sitio a cielo abierto en Patagonia.

Desde una perspectiva arqueométrica, Reigadas (1992, 2014, entre otros), abordó el estudio microscópico de pelos, fibras y pieles tanto para sitios de Patagonia como del NOA. La metodología de análisis desarrollada por la autora le ha permitido llevar a cabo tanto la identificación taxonómica de las fibras, como la construcción del perfil etario, la inferencia de la estacionalidad de los sitios y el abordaje de aspectos climáticos. Asimismo, ha abordado el uso de las fibras como materia prima y el análisis de las diversas etapas de trabajo antrópico registradas sobre las pieles (e.g. curtido, pelado, pintado, cocido, etc). Así, el estudio de adherencias y trazas inorgánicas le permitió la identificación de pinturas sobre las pieles (Reigadas 2014).

En La Pampa, Berón y colaboradores (2012) abordaron desde una perspectiva interdisciplinaria, el estudio de un envoltorio funerario de cuero de uno de los entierros del sitio Chenque I (Lihuel Calel). En el trabajo se reparó tanto en la identificación taxonómica del cuero -que permitió determinar su pertenencia a Rheidae- como el estudio de los procesos de modificación antrópica que fueron instrumentados y permitieron su conservación.

En los últimos años, Marchione (2005, 2009) partiendo de la escasez de trabajos específicos ha sistematizando la información disponible y estableció un procedimiento metodológico para el estudio de los cueros arqueológicos, tomando como caso de estudio los artefactos del sitio Campo Moncada 2 ubicado en la provincia de Chubut (Marchione 2009; Marchione y Belleli 2013). La autora desarrolló un protocolo que aborda todas las etapas de trabajo de gabinete, partiendo desde la estabilización inicial y conservación de los materiales hasta el relevamiento de todos los aspectos tecno-morfológicos (Marchione 2009).

\section{Los sitios arqueológicos}

El sitio Don Isidoro 2 se ubica al sur del departamento 
Loventué ( $36^{\circ} 50^{\prime} 12^{\prime \prime}$ S y $65^{\circ} 14^{\prime} 22^{\prime \prime}$ O, en adelante DI2) y fue hallado en una formación medanosa actualmente edafizada con la vegetación típica del espinal o monte de caldén. Entre 1997 y 1998 se efectuaron dos campañas arqueológicas durante las cuales se excavó una superficie de $12,50 \mathrm{~m}^{2}$. El sitio se ha vinculado con un asentamiento de indígenas ranquelinos, correspondiente a los momentos en que se produjo el avance de las tropas militares por el territorio del cacique Baigorrita, poco antes de su muerte en 1879 (Tapia 2008).

El Fortín La Perra (L.S. 36 53' 25.3" y L.O. 65 15' 23", paraje El Carbón, departamento Loventué, en adelante FLP), fue un asentamiento militar instalado en la cúspide de un cerro de tosca de 350 m s/m. Entre 1999 y 2001se excavaron $30 \mathrm{~m}^{2}$ y se estima que el lugar estuvo habitado por una pequeña guarnición de soldados (Ministerio de Guerra y Marina, 1883-1885).

\section{Materiales y métodos}

El conjunto asciende a 83 fragmentos de cuero, de los cuales el $76 \%(n=63)$ corresponden al sitio DI2 y el $24 \%$ $(n=20)$ a FLP. La totalidad de este conjunto fue sometido a un acondicionamiento como una medida preventiva para detener el deterioro (Marchione2005). En segundo lugar, para dar cuenta de los procesos de confección, la funcionalidad y los agentes tafonómicos que afectaron a los fragmentos de cuero, se registraron las características tecno-morfológicas aplicando variables y categorías de análisis definidas por Marchione (2009):

-Tipo de artefacto: fragmento de cuero con pelo; fragmento de cuero sin pelo; tira de cuero; cordel tendón; tiento; etc.

- Rastros de trabajo: costuras; orificios; bordes recortados, marcas de raspado, rastros de pintura, entre otros.

-Dimensiones: alto, largo y espesor.

-Forma: Irregular, Triangular, Cuadrangular, Circular, Trapezoidal, Indeterminada.

-Bordes: rectos, quebrados, deshilachados, irregular, doblados.

-Marcas en la superficie: corte, rayas, pintura, impronta, otros.

- Flexibilidad: flexible, semiflexible, rígida, indeferenciado. -Estado de conservación: Muy malo, Malo, Regular y Bueno.

Los datos fueron registrados en planilla de cálculo de Excel que permitirá el análisis comparativo bajo la aplicación de filtros y tablas dinámicas. Aunque algunos de los fragmentos remontan y corresponden al mismo objeto aunque fueron registrados individualmente.

Partiendo de la premisa que en general las pieles provenientes de diversas especies tienen diferentes características de estructura celular y fibrosa, es posible su identificación taxonómica. Para ello es necesario la caracterización de los componentes en los que se dividen: capa flor, corium y lado carne. La capa flor se configura como la capa papilar de la dermis y es la zona más externa de la piel, donde se conservan las paredes de los folículos pilosos y generalmente restos de pelo en su interior. A través de dicha capa es que se realiza la identificación taxonómica con el método propuesto. El corium es la parte subyacente a la capa flor y representa la capa reticular de la dermis. El lado interno de las muestras es el denominado "lado carne" del cuero y es la superficie que limita con el tejido subcutáneo o hipodermis en la piel original (de Perinat 2000; Markán 2015). De esta manera, en una tercer etapa se, se llevó a cabo el estudio taxonómico de las pieles por el personal del Centro de Investigaciones y Tecnologías del Cuero (CITEC- INTI), a cargo del Ing. Markán. Para ello se seleccionó una muestra inicial de 22 fragmentos, a los que se sumaron en un segundo momento 10 cueros. Para el total de los 32 fragmentos se tomó como criterio para su selecciónaquellos fragmentos que mostraron un mejor grado de conservación. Asimismo, como se mencionó anteriormente muchos fragmentos correspondían a una misma pieza, por ello en esos casos se seleccionó uno o más fragmentos (según el caso), como representación de la totalidad, optando por preservar el resto de las muestras. De cada muestra se efectuaron cortes delgados y fueron analizados con Microscópio óptico Olympus $\mathrm{CH} 2$ (en adelante MO). Para observar otros rasgos de las piezas se utilizó una lupa binocular estereoscópica Olympus SZ61 con cámara Infinity adosada (en adelante LBE).

Para la observación de las muestras en la LBE se debió realizar la limpieza del material con pincel y gasa para eliminar todo resto de sedimento, evitando dañar la muestra. Luego, se cortaron fragmentos perpendiculares a la superficie, lo que permitió observar la disposición de los tejidos a través del espesor e identificar si los cueros corresponden a especies de vertebrados mamíferos. De corroborarse este origen, se procedió a la diferenciación de las denominadas capa flor y capa corium. Para el análisis bajo $\mathrm{MO}$, se tomaron secciones de las muestras que fueron fijadas en solución de Sandison durante una semana. Luego se prosiguió según la técnica de inclusión en parafina hasta la obtención del taco para corte. Se llevaron a cabo cortes con micrótomo $(15 \mu-20 \mu$ de espesor). A su vez los cortes fueron desparafinados, aclarados en xilol y montados con bálsamo de Canadá sin efectuarles ninguna tinción. Los preparados fueron observados en $\mathrm{MO}$ en busca de restos de paredes de folículos pilosos y características de los mismos para poder asociarlos a un origen específico. Las muestras que a la LBE se vieron formadas únicamente por el espesor correspondiente a la capa corium se dejaron fuera de este procedimiento dado que no permiten realizar inferencias sobre la pertenencia taxonómica de los cueros.

Finalmente, los datos arqueológicos fueron interrelacionados con diversas fuentes documentales consultadas en los repositorios del Servicio Histórico del Ejército (SHE) y del INDEC, así como en las bibliotecas 

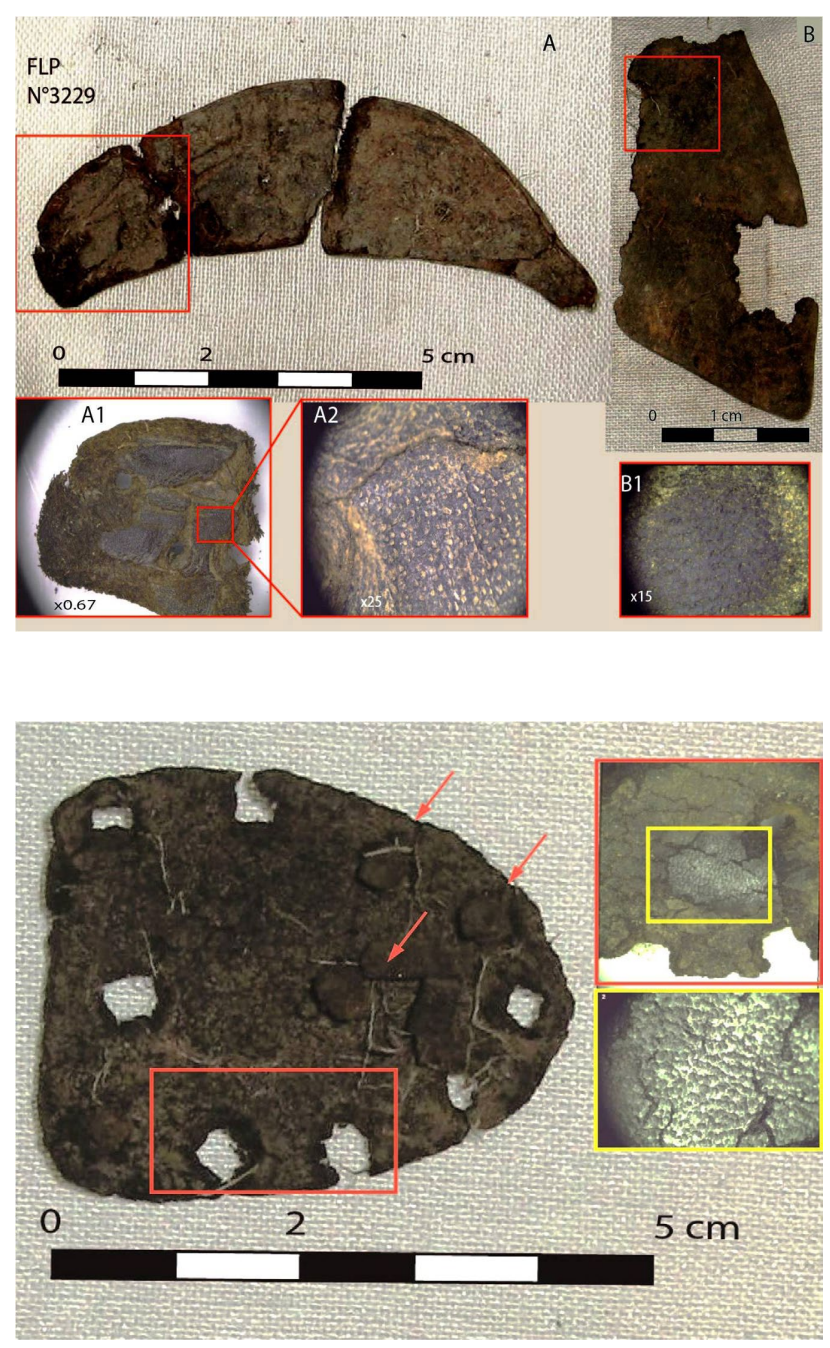

Figura 2. Suela de cuero del sitio DI2 de Camelidae. Se señalan con flechas la conservación de clavos de sección cuadrangular. Se amplía el detalle con lupa binocular x0.67 y x25 de la superficie del patrón flor.

Figure 2. Leather sole of the Camelidae site DI2. The arrows indicate the conservation of nails of quadrangular section. The detail of the quadrangular holes is enlarged with binocular loupe $\times 0.67$ y $\times 25$ of grain layer.

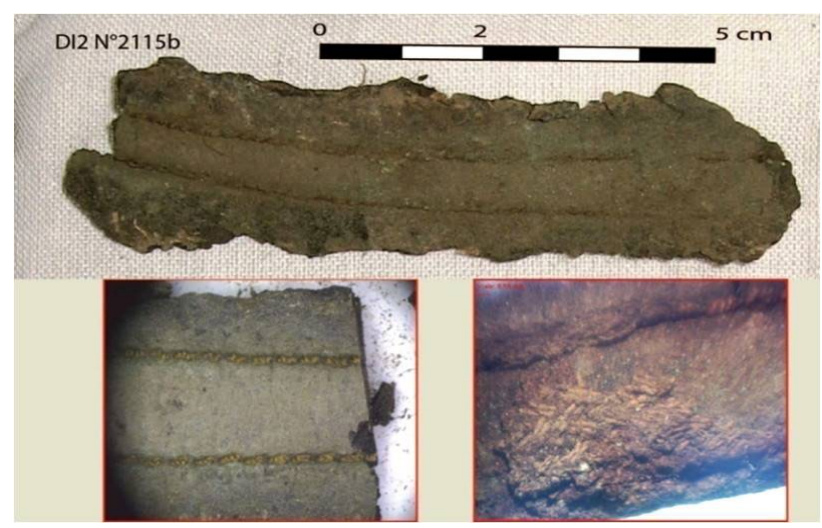

Figura 3. Fragmento de correaje con costuras (arriba y a la izq.) y textil adherido (der.)

Figure 3. Straps fragments with stitching (up left) and attached fabric (rigth).
Figura 4. Cuero de Bos taurus con ojales circulares. A1, detalles del cuero limpio y ojales delimitados (Imagen con lupa binocular $\times 0.67) ; A 2$, detalle de patrón de los folículos pilosos en la superficie flor (Imagen de lupa binocular x25); B, cuero de Camelidae con impronta de aplique cuadrangular; B1 detalle del patrón flor (imagen de lupa binocular x15).

Figure 4. Bos taurus leather with circular eyelets. A1, details of clean leather and delimited eyelets (Image with binocular loupe x0.67); $A 2$, detail of hair follicles patterns on flower surface (Image of binocular loupe x25); B, Camelidae leather with quadrangular appliqué imprint.

del Estado Mayor del Ejército y del Círculo Militar. En los dos primeros casos se relevó una serie de documentos primarios como la Colección "Frontera con el indio" (SHE) y fuentes secundarias como las Memorias de Guerra y Marina (MGM) del período 1860-1885, los reglamentos de Uniformes y los registros de Aduana. Asimismo, se utilizaron las memorias de viajeros y militares que han plasmado en sus relatos aspectos de la vida cotidiana en el espacio fronterizo (Prado 1960; Armaignac 1974; Daza 1975; Guerrino1984; Avendaño 2000; Mansilla 2006; Seymour 2013). Su análisis será presentado en la discusión en relación a las evidencias arqueológicas.

\section{Resultados}

El conjunto mostró un grado de conservación de regular a malo, tal como lo indica el aspecto de los fragmentos resquebrajados y rígidos y en su mayoría con los bordes irregulares. Los materiales del sitio DI2 poseen evidencias de un mayor deterioro. Asimismo, los cueros muestran un alto grado de fragmentación de los materiales, inferido a partir del remontaje de varias piezas como parte de un mismo objeto. En general el conjunto proveniente de ambos sitios posee marcas de radículas y sedimentos adheridos, los que no fueron removidos para evitar un mayor daño sobre los materiales (Tabla 1).

Respecto al tipo de artefactos registrados en DI2, el análisis tecnomorfológico permitió determinar que el 95\% del conjunto es atribuible a una función específica: un total de 15 fragmentos corresponden a correajes o fragmentos de aperos todos con similares características, por lo que podrían corresponder al mismo objeto y/o función. Asimismo, se registraron 45 fragmentos correspondientes a un calzado (suela, plantilla y capellada) y 3 fragmentos de cuero sin pelo (no identificadas, en adelante NID). En 9 fragmentos se observaron orificios cuadrangulares alineados producidos por los clavos que fijan las suelas del calzado. En algunos fragmentos aún se conservan adheridos los clavos de sección cuadrangular, los que fueron apartados para la realización del correspondiente análisis metalográfico (Figura 2). En los demás casos se hallan evidencias de costura industrial y presencia de fragmentos textiles y manchas de óxido de algún metal que formó parte de un correaje o similar (Figura 3). Para 
Tabla 1. Características tecnomorfológicas de los cueros analizados. Se detallan las muestras analizadas en el CITEC para su identificación (ID) taxonómica. NOTA= Ra: Raíces; OX: óxido

Table 1. Technomorphological characteristics of analyzed leathers. The samples analyzed in the CITEC are detailed for their taxonomic identification (ID)

\begin{tabular}{|c|c|c|c|c|c|c|c|c|}
\hline Sitio & $\mathrm{N}^{\circ}$ inv & Tipo de artefacto & $\mathrm{N}$ & $\begin{array}{c}\text { Rastros de } \\
\text { trabajo }\end{array}$ & Largo $\mathrm{x}$ ancho $(\mathrm{mm})$ & Marcas & Conservación & $\begin{array}{l}\text { Muestra ID } \\
\text { taxonómica }\end{array}$ \\
\hline FLP & 3367 & Cinto/correa & 1 & Costura industrial & $60 \times 24$ & RA & Malo & $n=1 \mathrm{NID}$ \\
\hline FLP & 3271 & Fragmento sin pelo & 4 & NID & $\begin{array}{l}54 \times 7 ; 50 \times 7 ; \\
22 \times 9 ; 23 \times 9\end{array}$ & RA & Malo & $\mathrm{N}=3$ Bos taurus \\
\hline FLP & 3325 & Fragmento sin pelo & 1 & Orificios & $29 \times 25$ & RA & Malo & $\mathrm{n}=1$ Camelidae \\
\hline FLP & 3226 & Fragmento sin pelo & 1 & NID & $69 \times 9$ & RA / OX & Malo & $n=1 \mathrm{NID}$ \\
\hline FLP & 3304 & Correa & 1 & Orificios & $84 \times 22$ & RA & Malo & $\mathrm{n}=1$ Camelidae \\
\hline FLP & 3244 & Fragmento sin pelo & 3 & $\begin{array}{l}\text { Orificios (1 } \\
\text { fragmento) }\end{array}$ & $30 \times 17 ; 15 \times 15 ; 15 \times 15$ & RA & Malo & $\mathrm{n}=3$ Camelidae \\
\hline FLP & $3322 a$ & Fragmento sin pelo & 4 & NID & $\begin{array}{c}60 \times 40 ; 40 \times 10 \\
36 \times 18 ; 15 \times 8\end{array}$ & RA & Malo & $\mathrm{n}=4$ Camelidae \\
\hline FLP & $3322 b$ & NID & 1 & $\begin{array}{l}\text { impronta } \\
\text { cuadrangular }\end{array}$ & $10 \times 7$ & RA & Malo & - \\
\hline FLP & 2451 & Fragmento sin pelo & 1 & NID & $50 \times 14$ & RA & Muy Malo & $\mathrm{n}=1 \mathrm{nid}$ \\
\hline FLP & 3229 & Calzado & 3 & $\begin{array}{c}\text { Orificios } \\
\text { cuadrangulares }\end{array}$ & $\begin{array}{c}37 \times 19 ; 27 \times 18 \\
18 \times 14\end{array}$ & RA & Malo & $\mathrm{n}=2$ Bos taurus \\
\hline $\mathrm{DI} 2$ & 577 & Fragmento sin pelo & 3 & NID & $30 \times 13 ; 22 \times 15 ; 5 \times 4$ & RA & Malo & - \\
\hline $\mathrm{DI} 2$ & 818 & Zapato (Suela) & 2 & $\begin{array}{l}\text { Orificios con } \\
\text { clavos }\end{array}$ & $35 \times 16$ & RA & Malo & - \\
\hline DI2 & 58 & Zapato (Suela) & 2 & $\begin{array}{l}\text { Orificios con } \\
\text { clavos }\end{array}$ & $35 \times 30$ & RA & Malo & $\mathrm{n}=2$ Camelidae \\
\hline DI2 & $\begin{array}{c}2115 \\
a\end{array}$ & correa & 1 & Costura industrial & $45 \times 24$ & RA / OX & Malo & $\mathrm{n}=1$ nid \\
\hline DI2 & $2115 b$ & correa & 2 & Costura industrial & $81 \times 21$ & RA / OX & Malo & $\mathrm{n}=1$ nid \\
\hline DI2 & $2115 c$ & correa & 1 & Costura industrial & $120 \times 13$ & RA / OX & Malo & - \\
\hline $\mathrm{DI} 2$ & $2115 d$ & correa & 11 & Costura industrial & $15 \times 14$ & RA / OX & Malo & $\mathrm{n}=3 \mathrm{nid}$ \\
\hline DI2 & $12 a$ & Zapato (Suela) & 9 & $\begin{array}{c}\text { Orificios } \\
\text { cuadrangulares }\end{array}$ & $255 \times 61$ & RA & Malo & $\mathrm{n}=4 \mathrm{nid}$ \\
\hline DI2 & $12 \mathrm{~b}$ & $\begin{array}{l}\text { Zapato (Suela y } \\
\text { capellada) }\end{array}$ & 32 & $\begin{array}{c}\text { Orificios } \\
\text { cuadrangulares }\end{array}$ & $65 \times 44 ; 25 \times 18$ & RA & Malo & $\begin{array}{l}\mathrm{n}=3 \text { Camelidae; } \\
\mathrm{n}=1 \text { Bos taurus }\end{array}$ \\
\hline
\end{tabular}

el caso de Fortín La Perra, 15 fragmentos corresponden al tipo de cuero sin pelo (NID), 3 corresponden a suela de calzado y 2 son tiras de cuero que pueden atribuirse a restos de correajes.

El $40 \%$ de los fragmentos $(n=8)$ muestra rastros de trabajo, siendo en un caso evidencias de costuras de tipo industrial (tira de cuero de un posible correaje). En 7 casos se observan orificios de costuras y en un fragmento se distingue la impronta de algún aplique o insignia cuadrangular (Figura 4).

En una primera instancia 14 muestras $^{1}$ (compuestas por 22 fragmentos), fueron analizadas en el CITEC para su identificación taxonómica. El 57,1\% de las muestras pudieron identificarse con algún nivel de especificidad: $14,2 \%$ a nivel de especie y $42,9 \%$ a nivel de familia (Tabla 2). El 42,9\% restante no pudo identificarse dada las condiciones de conservación. Los restos identificados a nivel de especie corresponden a dos muestras provenientes del sitio FLP identificadas como Bos taurus

\footnotetext{
${ }^{1}$ Cada muestra estaba representada por uno o más fragmentos correspondientes a una misma unidad.
}

Tabla 2. Restos identificados taxonómicamente. Frecuencias absolutas y relativas. Nota: NID= no identificados; NISP= especímenes identificados

Table 2. Remains identified taxonomically. Absolute and relative frequencies.

\begin{tabular}{lll}
\hline Taxón & NISP & $\%$ \\
\hline Bos taurus & 2 & $14,3 \%$ \\
Camelidae & 6 & $42,9 \%$ \\
NID & 6 & $42,9 \%$ \\
\hline
\end{tabular}

(vaca) a través del patrón de aberturas foliculares y su agrupamiento observados en la capa flor (folículos simple) (Figura 4A). Uno de los fragmentos de cuero vacuno identificados correspondería a un fragmento de suela de calzado -asociada a un fragmento tejido de origen vegetal. El otro corresponde a un fragmento con función no determinada. Los restos identificados a nivel de familia corresponden a Camelidae (camélido). $\mathrm{Si}$ bien esos fragmentos no han podido identificarse a una función determinada, poseen rastros de confección 
(Figura 3). Respecto a los restos correspondientes a Camelidae hallados en DI2 se trata de fragmentos de suela y capellada de calzado.

La identificación taxonómica de la familia Camelidae en algunos fragmentos de cuero y su vinculación con las características tecno-morfológico (correaje y calzado), constituyen resultados singulares cuyas implicancias se discuten en el siguiente ítem. Al respecto, con el fin de precisar los resultados del análisis realizado en el CITECINTI, se solicitó asesoramiento al Dr. Eduardo Frank, especialista en cueros de camélidos americanos de la Facultad de Ciencias Agropecuarias, de la Universidad Católica de Córdoba. A través del estudio de las imágenes él corroboró la identificación taxonómica (Eduardo Frank, comunicación personal 2017).

Teniendo en cuenta los resultados obtenidos inicialmente, se seleccionaron 10 nuevas muestras de los materiales con taxón NID y Camelidae con el fin de repetir los análisis para precisar la especie. Un total de cuatro fragmentos correspondientes a DI2 y seis a FLP se analizaron nuevamente en el CITEC, aunque los resultados no han arrojado nuevos datos, exceptuando el caso de un fragmento de calzado hallado en DI2 que corresponde a la capellada. Dicho fragmento fue identificado como cuero vacuno, siendo diferente a la asignación taxonómica de la suela de la misma pieza, confeccionada con cuero de camélido. De acuerdo con estos resultados, cabe preguntarse si fue posible que la suela y la capellada hayan sido fabricadas a partir de diferentes materias primas o si se trata de calzados diferentes. Otra posibilidad es que el cuero vacuno haya sido utilizado como parche para reparar el calzado original, aunque las malas condiciones de toda la pieza hacen imposible verificar esta inferencia.

\section{Discusión y conclusiones}

Los fragmentos de cuero recuperados en FLP y DI2 habrían formado parte de calzados y correajes, tanto de uniformes militares como de vestimenta civil o aperos. La presencia de uniformes militares en las tolderías indígenas no resulta un hecho extraño, dado que dichas prendas eran comúnmente obsequiadas o intercambiadas, tal como se registran en diversas fuentes documentales (Ávila 2002; Mansilla 2006; Zavala y Tamagnini 2011) y fue ratificado por el hallazgos de otras evidencias materiales como botones militares (Landa 2011). Por otra parte, la identificación taxonómica de los cueros arqueológicos se puede correlacionar con diversas menciones que proporcionan las fuentes documentales (relatos de viajeros y documentación oficial) respecto al uso de cuero vacuno, ovino y/o equino para diversos usos y funciones (Memoria de Guerra y Marina 1860-1885; Ejército Nacional 1871; entre otras). En el análisis de las fuentes documentales, Tapia (2008) ha registrado que el cuero fue una de las materias primas más utilizadas entre los indígenas y militares de la pampa seca, siendo utilizado mayoritariamente para la confección de elementos ecuestres (maneas, bozales, lazos, pellones, etc.); enseres de cocina (tipas, ñoques, alforjas); mobiliario (cama, toldos, asientos, correas para fijar las estructuras de madera, mantas, etc.) y vestimenta (botas, sombrero, etc.).

A su vez, en el marco del espacio fronterizo se ha destacado recurrentemente el uso de "cuero de potro" para la confección de calzados (Armaignac 1975; Daza 1975; Guerrino1984; Assuçao1992; Avendaño 2000; Seymour 2013; entre otros). También se aludió al uso de cuero ovino y vacuno para realizar calzados y aperos, como también para la construcción de viviendas, refugios y tolderías (Servicio Histórico del Ejército, 1860, 1862, 1865,1867, 1870; Udaondo 1922; Luqui- Lagleyse1995; D’Orbigny1999; Avendaño 2000; Mansilla2006).

También se mencionó el uso de cuero vacuno para la confección de sogas (Daza 1975; Avendaño 2000) y el de animales silvestres (guanacos, zorros, pumas, vizcachas, ñandú, etc.) tanto para la fabricación de aperos y vestimenta como para su comercialización, dado su valor de intercambio entre indígenas, militares y criollos.

La búsqueda de información sobre el uso de cueros en los documentos del MGM se orientó a la categoría vestimenta, la cual está relacionada con la importación de uniformes -incluyendo el calzado - y telas desde Europa (1863, 1864, 1865, 1866 y 1868) y con la confección de uniformes para el ejército nacional en los talleres de la Comisaría General de Guerra y Marina (1875 y 1877). En la documentación que incluye esta colección se menciona que, en una primera etapa, se propició la importación de telas y/o la confección de los uniformes en Francia; mientras que, en una segunda etapa, se enfatizó su producción nacional en los talleres del Ministerio de Guerra y Marina. De este modo, en el año 1864, en una carta al ministro de Guerra y Marina Gelly y Obes se presentó el proyecto de Ley enviado al Congreso Nacional para la importación desde Francia de los vestuarios y calzados del ejército. A partir de la calidad de estos uniformes, que emularían a los del ejército francés, se estipuló una reducción de los costos debido a que la durabilidad de cada muda sería mayor. Así se propuso que "(...) en vez de tres camisas, é igual número de calzoncillos, sólo se toman dos de cada clase; y en el calzado, en lugar de cuatro pares, tres; todo en razón de la mejor calidad de los artículos que garanten igual o mayor duración" (MGM 1863-1865:86).

Tal como se expresa en la cita, los calzados también fueron importados como parte del uniforme. La reducción en la frecuencia de reposición podría explicar el hallazgo recurrente de los restos de calzados en los sitios arqueológicos, producto del desgaste luego de largos períodos de tiempo sin ser reemplazados. Sin embargo, sigue siendo materia de especulación 
la procedencia de la materia prima utilizada para su fabricación. En relación a la compra de los uniformes en Francia, si bien en la documentación se menciona que las gestiones las debía realizar de modo directo el Gobierno Nacional, a partir de su representante en suelo francés, se observa que las compras se realizaron por intermediarios. De esta manera, se constató que "La Nación costea así el provecho pecuniario de los intermediarios, que a veces venden de segunda y de tercera mano, según la situación de plaza" (MGM 1878: VI). Para los años 1869 y 1870 se registró en el listado de compras la diferenciación entre "botas del país" y "botas extranjeras", las que representan porcentajes similares respecto al volumen total adquirido y al valor pagado por cada par. La tercerización en la compra de elementos dificulta conocer qué tipos de artículos fueron efectivamente provistos, de acuerdo a su composición, calidad y procedencia. Asimismo, obstaculiza saber si se han cumplido las normas materiales y de calidad que estipulaba el Ejército Nacional, reglamentando que los calzados y polainas de cuero debían ser confeccionados a partir de cuero de vacuno o becerro en color negro (Ejército Argentino 1871).

A partir del año 1875, se encontraron repetidas menciones respecto a los crecientes esfuerzos para la confección de los uniformes en talleres de la Comisaría General de Guerra y Marina -aunque los géneros y otras partes constituyentes como botones o bramantes continuaron importándose. Entre tanto -previa licitación- se efectuó un contrato con la fábrica de paños de la ciudad de Buenos Aires para la entrega de telas al Ejército. De este modo, se intentaba proteger la industria nacional, a la vez que obtener una clase de paño superior al que se usaba hasta ese momento en el vestuario (MGM, 1875:19).

En torno a los elementos de cuero como correajes y aperos se mencionó a menudo su mal estado y la reparación de los mismos a partir de cueros de vacas y ovejas comprados a los proveedores: "En las monturas se han invertido gran número de cueros, tanto vacunos como lanares, para componerlas siendo urgente sustituirlas por otras nuevas. Los correajes, aún más servibles, no dejan por esto de reclamar su próximo reemplazo..." (MGM 1878:87).

En las listas de almacenes e inventarios publicados en las MGM es corriente la referencia a elementos del vestuario, aperos y equipamiento, registrándose 21 elementos de cuero, con una amplia variabilidad en torno a las correas, existiendo 12 tipos diferentes de acuerdo a su función (Figura 5).

A partir del análisis de la documentación consultada en el SHE se observa que los cueros de las reses racionadas -aparte de la carne- debían abonarse al proveedor, por lo que su uso implicó la previa autorización de la Contaduría General del Ejército. Estos pedidos se registraron de forma recurrente (años 1860, 1861, 1862, 1863, 1867 y 1876) para la confección de bozales, maneadores y correas en reemplazo de las existentes en pésimo estado. Para ello, la Comisaria de Guerra había calculado que de un cuero de vaca o novillo era posible confeccionar doce maneadores, veinticinco bozales y entre ochenta y cien maneas. En junio de 1865, se presentó la solicitud para la construcción de toldos para la tropa del Campamento del Perdido, sin embargo dicho documento se ha extraviado, por lo que no se conocen mayores detalles. A partir de estas referencias, se podría inferir que en

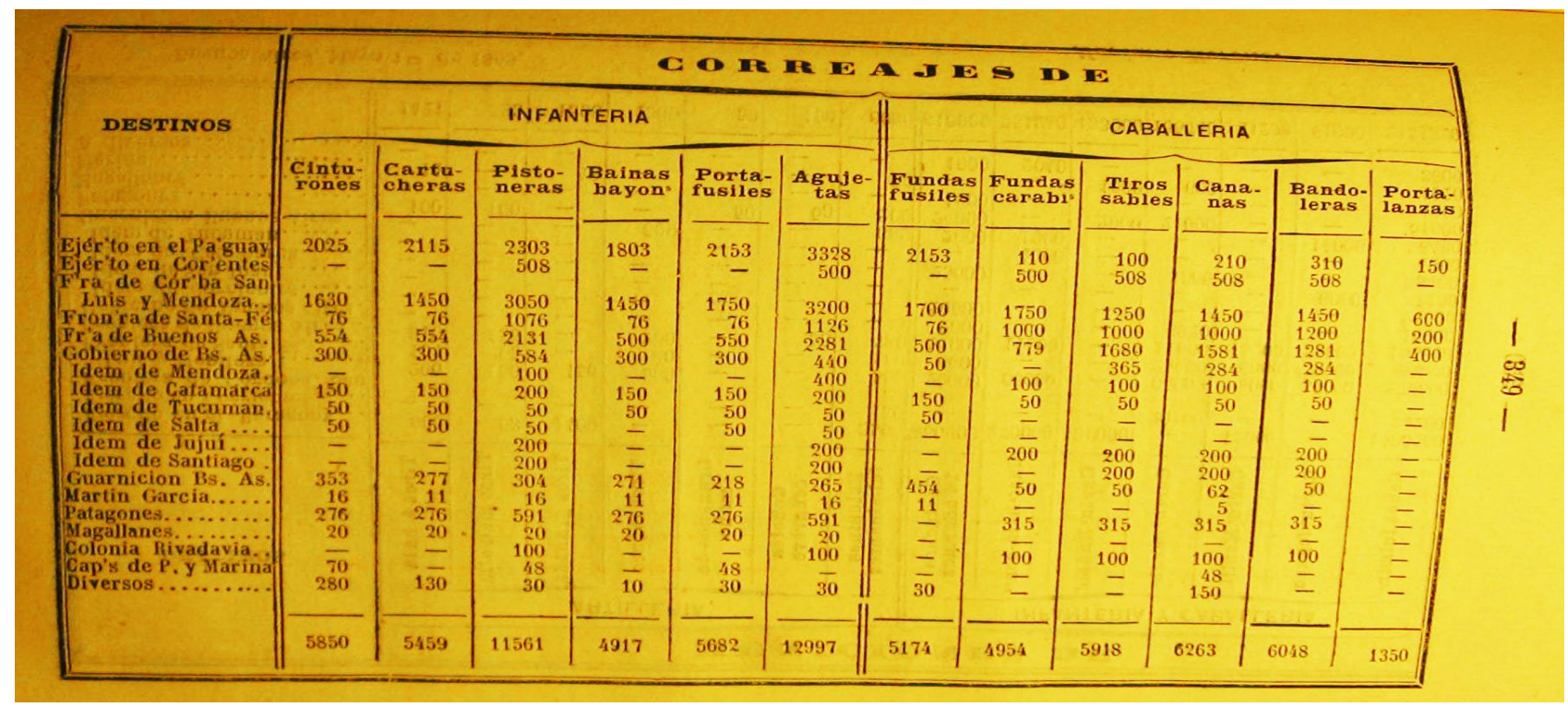

Figura 5. Tabla con detalle de los correajes correspondiente a los uniformes militares y aperos (Memorias de Guerra y Marina 1869:349).

Figure 5. Table detailing the straps corresponding to military uniforms and the tack (Memorias de Guerra y Marina 1869: 349). 
Tabla 3. Exportaciones e importaciones de cueros de camélidos, vestuario y equipamiento militar. Nota: N/D= no hay dato.

Table 3. Exports and imports of camel hide, clothing and military equipment.

\begin{tabular}{|c|c|c|c|c|c|c|c|c|c|c|}
\hline \multirow[b]{3}{*}{ Año } & \multicolumn{2}{|c|}{ Exportación } & \multicolumn{8}{|c|}{ Importación } \\
\hline & Bélgica & N/D & Francia & & Inglate & & Brasil & & Bélgica & N/D \\
\hline & 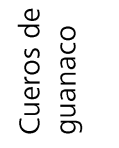 & 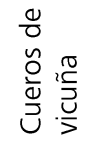 & 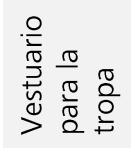 & 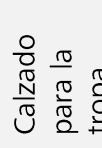 & 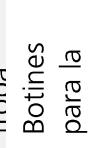 & 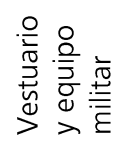 & 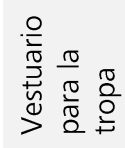 & 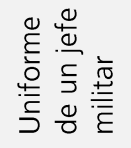 & 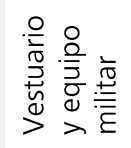 & 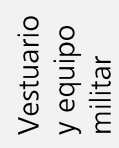 \\
\hline 1862 & - & - & $x$ & - & - & - & $x$ & $x$ & - & - \\
\hline 1863 & $x$ & - & $x$ & - & - & - & - & - & - & - \\
\hline 1864 & - & - & - & $x$ & - & - & - & - & - & - \\
\hline 1865 & - & - & - & - & - & - & - & - & - & - \\
\hline 1866 & - & - & $x$ & $x$ & $x$ & - & - & - & - & - \\
\hline 1867 & - & - & & - & - & - & - & - & - & - \\
\hline \multicolumn{11}{|c|}{ Periodo 1868- 1872: N/D } \\
\hline 1873 & - & - & $x$ & - & - & $x$ & - & - & $x$ & - \\
\hline 1874 & - & - & $x$ & - & - & $x$ & $x$ & - & - & - \\
\hline 1875 & - & - & $x$ & - & - & $x$ & - & - & $x$ & - \\
\hline 1876 & - & - & - & - & - & - & - & - & - & $x$ \\
\hline 1877 & - & - & - & - & - & - & - & - & - & - \\
\hline 1878 & - & - & - & - & - & - & - & - & - & $x$ \\
\hline 1879 & - & - & - & - & - & - & - & - & - & $x$ \\
\hline 1880 & - & $x$ & - & - & - & - & - & - & - & - \\
\hline Periodc & $1881-1$ & 85: N/L & & & & & & & & \\
\hline
\end{tabular}

los asentamientos existió el laboreo del cuero para confeccionar y reemplazar los elementos en mal estado, entre algunos de los usos registrados.

El análisis de los registros de Aduana brindó algunos datos de importancia, a pesar de los sesgos que presentó el faltante de algunos libros. La exportación de cueros de camélidos sudamericanos (guanaco y vicuña) fue declarada en dos ocasiones, la primera en 1862 con destino a Bélgica y la segunda en 1880 sin especificar su destino (Tabla 3). Dentro de los cueros exportados existió la categoría de "Diversas pieles" que se mantuvo constante a lo largo de los años y tuvo múltiples destinos, principalmente europeos. Es probable que dentro de esa categoría se hayan incluido cueros de camélidos sudamericanos, comercio que tuvo su auge durante el siglo XVIII y prosiguió en el siglo XIX. No obstante, dicha actividad fue prohibida desde momentos tempranos debido a la sobreexplotación de los animales especialmente en el Noroeste argentino y por este motivo se explicaría su omisión en los registros oficiales (Yacobaccio et al. 2003; Benedetti y Conti 2009).

Respecto a la importación, dentro de los bienes libres de impuestos se observaron las categorías de "vestuarios hechos para la tropa", "calzado para la tropa" o "vestuario y equipamiento militar". Los calzados fueron importados en los años 1864 y 1866 provenientes de Francia e Inglaterra. Por su parte, los vestuarios y equipamientos, procedían principalmente de Francia, aunque también se registró su importación desde Inglaterra, Bélgica y Brasil. Estos artículos ingresaron en los años 1862, 1863, 1866 y en el período comprendido entre 1873 y 1879. En comparación con los datos obtenidos de las MGM, existe coincidencia para el período 1863-1866 en relación a la importación de uniformes. Sin embargo, durante la década de 1870 los registros de Aduana presentan datos en parte contradictorios sobre la confección de uniformes en los talleres de la Comisaría de Guerra (MGM 1875, 1878). Por ejemplo, se indica la importación de vestuarios militares de diversos países, pero Francia ha sido el país más representado, contradiciendo otros documentos oficiales.

Dado que el análisis de los cueros arqueológicos realizados en el CITEC indican que algunos de los calzados y otros elementos no identificados fueron confeccionados con cueros de camélido, el origen de los mismos se constituye como un aspecto significativo a dilucidar. En primer lugar, los cueros de Camelidae identificados 
poseen claras evidencias de su elaboración industrial, hecho que suscita diferentes interrogantes. Por otra parte, las fuentes documentales no presentan datos que den cuenta del uso de cuero de camélidos sudamericanos (silvestres o domesticados) para la producción a nivel industrial durante el siglo XIX en nuestro país o la región. En efecto, considerando el fácil acceso y abundancia de los cueros de vacunos sería bastante atípico que para la fabricación industrializada de calzado se haya utilizado cuero de guanaco, vicuña o alpaca. La importación de los uniformes desde Francia se registró en las MGM a partir del año 1864 (MGM1864; Udaondo 1922; Luqui Lagleyse 1995; Leoni 2009). A su vez, el análisis de los libros de Aduana indica la importación de vestuario, calzados y equipamiento para la tropa proveniente de Francia y de otros países (Inglaterra, Bélgica y Brasil), para diversos momentos de la década de 1860 y 1870 (Tabla 3).

Si bien los datos de esas importaciones indican que fueron realizadas entre 20 y 13 años antes a la ocupación de los sitios bajo estudio, la existencia de rezagos en depósitos y almacenes militares podría explicar su presencia en el registro arqueológico pampeano. Así, el hallazgo en FLP de un botón con la inscripción en su reverso "Superieur France" remite a su origen francés, abonando la hipótesis de la presencia de uniformes importados de Francia en ese asentamiento (Landa 2011). Dicho botón correspondería a un uniforme de alto rango, según los datos que ha brindado el análisis documental y arqueometalúrgico (Figura 6). No obstante, sigue siendo de interés identificar la procedencia de los cueros de camélidos para la fabricación de los hallazgos de cuero, por lo que planteamos dos inferencias alternativas:

1- durante la primera mitad del siglo XIX se realizaron algunas exportaciones de cueros de guanacos, vicuñas o alpacas a Europa, en este caso los calzados militares comprados en Francia bien pudieron ser elaborados con los cueros de los camélidos sudamericanos (Yacobaccio et al. 2003);

2- es sabido que en la última mitad del siglo XIX Francia importaba cueros de dromedario de sus emplazamientos coloniales en Argelia, por este motivo se podría haber utilizado esa materia prima en la confección de piezas de los uniformes militares, que luego compró el Ejército argentino para la campaña del desierto entre 1876-1878 (Ximénez Sandoval y Madera y Vivero 1853).

En relación a la primera de las alternativas, es relevante mencionar que se produjo la exportación de cueros y fibras de vicuña y guanaco hacia Europa desde el período colonial hasta mediados del siglo XIX (Yacobaccio et al. 2003; Benedetti y Conti 2009). Sin embargo, esta actividad no fue oficialmente declarada para fines del siglo XIX. Por lo tanto, en los registros de Aduana solo en dos ocasiones se mencionó la exportación de cuero de camélidos. En relación a la segunda opción, varias referencias directas e indirectas dan cuenta de la compra

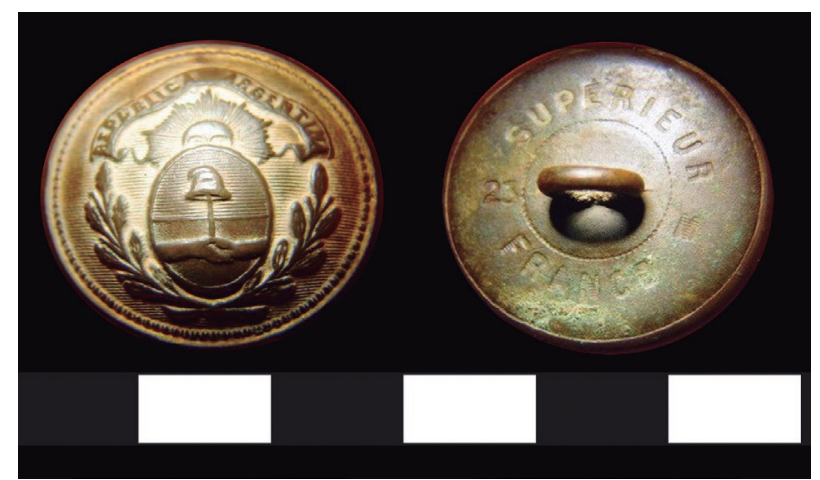

Figura 6. Botón de uniforme militar hallado en FLP. En su anverso se distingue el Escudo Nacional y en el reverso se observa la inscripción en relieve "Superiuer France 23.

Figure 6. Military uniform button found on FLP. On its obverse, the National Coat of Arms can be seen and on the back, there is an inscription in relief: Superiuer France 23.

de piezas militares de cuero a Francia.

Terminada la guerra de Crimea, el año 1856, el gobierno del Estado de Buenos Aires adquirió, por medio de su representante diplomático en Francia, uniformes para el ejército, por ser un precio más ventajoso que los hechos en el país y de más duración (Udaondo 1922:210)

Asimismo, desde Argelia se exportaron insumos para la confección de productos manufacturados en Francia (Ximenez de Sandoval y Madera y Vivero, 1853: 109). Se ha indicado el uso de cuero marroquí (de dromedario) como parte del uniforme de las fuerzas regulares en Argelia: "Por armamento y correaje dioles una cartuchera de cuero marroquí, suspendida por una correa estrecha, y sujeta con otra de cinturón al lado derecho; un fusil con bayoneta..." (Ximenez de Sandoval y Madera y Vivero, 1853: 323). Sin embargo, no se han hallado hasta el momento referencias acerca del uso de este tipo de cueros en la industria manufacturera francesa para el siglo XIX.

A pesar de ello, la denuncia de irregularidades en torno a la importación de los uniformes de Francia (u otros países europeos), sostiene la posibilidad de inferir alguna maniobra poco transparente por parte del intermediario que proveía al Ejército Nacional: "Los uniformes para la infantería, llegaron hechos y se expusieron al público como confeccionado en el país, y como fuera descubierta la etiqueta, dio origen a críticas en los periódicos opositores al gobierno" (Udaondo1922:210). Este escenario toma mayor relevancia a la luz de las incongruencias halladas entre las MGM y los Libros de Aduana, correspondientes a los años en que los uniformes fueron importados y a los años en que se produjeron en el país.

En cuanto a la producción artesanal y local de artefactos de cuero, fue mencionada en diversas fuentes documentales tanto para sitios indígenas como militares (SHE 1860-1885; 
Racedo 1940; Avendaño 2000; entre otros). Respecto a su evidencia material indirecta, en los sitios pampeanos se han hallado sobadores de piedra comúnmente utilizados para el laboreo del cuero (Tapia 2008). Dicha evidencia también fue hallada en asentamientos bonaerenses como Fortín Miñana y Otamendi (Gómez Romero 2007). Particularmente en el sitio DI2 se han hallado 5 artefactos líticos con evidencias de uso como sobadores. La presencia de raspadores también permite dar cuenta del trabajo de las pieles animales. Por último, el hallazgo de un posible punzón confeccionado a partir de un asta de Ozotoceros bezoarticus (venado de las pampas) y el punzón hallado en la colección de Fortín Toay (Vigne), también permitió pensar en su uso para el trabajo del cuero (Tapia 2008). A pesar de esas evidencias indirectas no se hallaron otros restos arqueológicos que expresen el desarrollo de dichas prácticas.

La imposibilidad de acceder a un mayor grado de especificidad en la identificación taxonómica no ha permitido zanjar la discusión hasta el momento. Más allá de las hipótesis planteadas, lo que se vislumbra en torno a la excepcionalidad del dato, es que la reglamentación de uniformes no fue respetada. El análisis arqueométrico de los elementos metálicos que componían el calzado quizás pueda arrojar nuevos datos para continuar profundizando la indagación.

\section{Palabras finales}

Dada la mala conservación y alta fragmentación del conjunto no ha sido posible realizar un análisis más exhaustivo sobre la función de los artefactos hallados. Solo se puede precisar que se trata de restos con rastros de trabajo antrópico industrial y no hay inicios de procesamiento artesanal o in situ. El mal estado conservación de los cueros ha imposibilitado la identificación a nivel específico en la gran mayoría del conjunto. Se han identificado algunos elementos pertenecientes a calzados y correajes que formaron parte de uniformes y aperos utilizados en la vestimenta militar durante la campaña del desierto. Su estudio ha permitido dar cuenta de las prácticas de consumo en el espacio fronterizo, donde primó la circulación de bienes industrializados, provistos y/o comerciados por sobre la producción local o artesanal.

Los resultados del estudio taxonómico, más allá de algunos especímenes pertenecientes a Bos taurus, ha proporcionado información novedosa en torno a la identificación de cueros pertenecientes a camélidos. A partir de los datos taxonómicos obtenidos, se plantea una serie de interrogantes formulados en base a la información documental que presenta datos contrapuestos respecto al origen y uso de los elementos de cuero. Sea cual fuese, está teñido de sospechas y denuncias en torno a la malversación de los fondos militares en relación a la provisión del ejército (Udaondo1922; Barros 1975). Como agenda de trabajo nos proponemos buscar nuevas vías de indagación arqueométricas, para resolver qué especie de camélido fue utilizada y cómo la misma fue introducida como bienes de manufactura industrial.

El estudio de los cueros arqueológicos ha brindado novedosa información al proceso de investigación sobre los sitios de frontera de la pampa seca, configurándose como una vía de indagación que no es usualmente aprovechada cuando se cuenta con ese tipo de materiales.

Buenos Aires, 6 de julio 2018

\section{Agradecimientos}

La investigación ha sido posible gracias al financiamiento del proyecto UBACyT dirigido por la Dra. Ana Margarita Aguerra y la Dra. Alicia Tapia. Se enmarcó en el proyecto de beca doctoral de Jimena Doval financiada por CONICET. Deseamos expresar nuestra gratitud al Ing. Markán y su equipo por la realización del estudio taxonómico de los cueros, a la Dra. Marchione por facilitarnos bibliografía y al Dr. Frank por asesorarnos en relación a los cueros de camélidos. A los evaluadores que con sus sugerencias han permitido mejorar el resultado final del artículo. Lo aquí vertido es completa responsabilidad de las autoras.

\section{Bibliografía}

Aguerre, A. M.2000.Las Vidas de Pati en la toldería de Río Pinturas y después. Tesis de doctorado inédita. Facultad de Filosofía y Letras, Universidad de Buenos Aires.

Armaignac, H. 1974[1883].Viaje por las pampas Argentinas. Eudeba, Buenos Aires.

Assucao, F.1992.Pilchas criollas. Editorial Emecé, Buenos Aires.

Avendaño, S.2000.Usos y costumbres de los indios de La Pampa. Editorial El Elefante Blanco, Buenos Aires.

Barros, A. 1975 [1872]. Fronteras y territorios federales de las pampas del sur. Solar Hachette, Buenos Aires.

Benedetti, A. y Conti,V. 2009. Explotación de los recursos naturales andinos: comercio y circuito de las pieles de chinchilla durante el siglo xIx y primeras décadas del xx. Si Somos Americanos. Revista de Estudios Tranfronterizos IX(2):117-140.

Berón, M., Di Donato, R.M. y Markán, J.A.2012.Leather funerary packages: Mortuary practices and differential preservation in a Late Holocene prehispanic cemetery (Pampean region, Argentina). Quaternary International278: 51-62.

Buc, N.2005. Análisis microscópico de instrumentos óseos del humedal del Paraná Inferior. Una primera aproximación experimental. A. Cetti, A. Re, D. Rindel y P. Valeri, Entre pasados y presentes, 262-279, INAPL, Buenos Aires.

Casamiquela, R. 2000. Temas patagónicos de interés arqueológico. VI. Análisis etnográfico de la morfología del toldo tehuelche y sus derivaciones etnológicas (hacia una "retro- 
etnología"). Intersecciones en Antropología1:3-33

Cattáneo, R. y Aguerre, A. 2009. Estudios funcionales de artefactos líticos de Cueva de las Manos, Río Pinturas, Santa Cruz, Argentina. Revista del Museo de Antropología 2: 3- 22.

Cattáneo, R. y Aguerre,A. 1996/1997. Rescate y Reinserción de los Mantos de Pieles (Quillangos) Indígenas de Patagonia en la Provincia del Chubut (Argentina).Cuadernos del Instituto Nacional de Antropología y Pensamiento Latinoamericano17:69-87.

Caviglia, S. 2002. El arte de las mujeres Aónikenk y Gununa Kuna. Las capas pintadas". Relaciones de la Sociedad Argentina de Antropología XXVII: 41- 70.

Ciampagna L, Hammond, H., Bogán, S., Trola, V., Aguinaga,M.C. y Zubimendi, M. 2006. Noticias sobre el primer hallazgo de artefactos de cuero en la Costa Norte de Santa Cruz, PatagoniaArgentina. X Congreso Nacional de estudiantes de Arqueología. CD-ROM.

Daza, J.1975 [1908]. Episodios militares. Eudeba, Buenos Aires.

Ejército Argentino. 1871. Reglamento propuesto por la Comandancia General de Armas prescribiendo el uniforme que debe usar el Ejército de la República aprobado por el Superior Gobierno y mandado poner en observancia. Imprenta El Nacional, Buenos Aires.

De Perinat, M.2000. Tecnología de la confección en piel. Edym, España.

Fernández Distel, A.2001. Calzado de los cazadores recolectores del noroeste argentino en la Colección arqueológica Torres Aparicio (Jujuy). Relaciones de la Sociedad Argentina de Antropología XXVI: 403- 414.

Guerrino, A.1984.La medicina en la Campaña del Desierto. Círculo militar, Buenos Aires.

Gómez Romero, F. 2007. Sistemas de relaciones sociales en la frontera sur de Buenos Aires: yacimientos Fortín Miñana (1860-1863) y Fortín Otamendi (1858-1869). Tesis doctoral inédita, Departamento de Prehistoria, Facultad de Filosofía y Letras, Universidad Autónoma de Barcelona.

Hermo, D. y Lynch, V. 2014. De la producción al uso: Tecnología de hojas en el sitio Cueva Maripe (Santa Cruz, argentina). IX Jornada de arqueología de la Patagonia.

Landa, C.2011. Fierros fronterizos: Los materiales de metal como indicadores de identidad y diferenciación social en la Frontera del Sur (1776-1885). Editorial Académica española, España.

Leoni, J.B. (2009). Armar y vestir al ejército de la nación: los artefactos militares del Fuerte General Paz. (Carlos Casares, Provincia de Buenos Aires) en el marco de la construcción del Estado nacional y la guerra de frontera. Intersecciones en Antropología 10:167-182.

Luqui-Lagleyze, J. M.1995. Del morrión al casco de acero. Los cuerpos militares en la historia argentina. Organización y Uniformes 1550-1950. Instituto Nacional Sanmartiniano, Comisión Argentina de Historia Militar y Fundación Mater-Dei,
Buenos Aires.

Mansilla, L. 2006[1881].Una excursión a los indios ranqueles. Editorial Emecé, Buenos Aires.

Marchione, P. C.2005. Tratamiento y Conservación de los cueros arqueológicos de Campo Moncada 2, Valle de Piedra Parada, Chubut. A. Pifferetti y R. Bolmaro, Metodologías Científicas aplicadas al Estudio de los Bienes Culturales, publicación del Primer Congreso Argentino de Arqueometría, Humanidades y Artes Ediciones, Rosario.

Marchione, P. C.2009.Análisis de los artefactos de cuero del sitio Campo Moncada 2, valle de Piedra Parada (Chubut). Tesis de Licenciatura inédita Facultad de Filosofía y Letras, Universidad de Buenos Aires.

Marchione, P. y Bellelli,C. 2013. El trabajo del cuero entre los cazadores-recolectores de la Patagonia centro-septentrional. Campo Moncada 2 (valle medio del Río Chubut). Relaciones de la Sociedad Argentina de Antropología XXXVIII: 223-246.

Markán, J.A.2015. Informe técnico N4799. CITEC. INTI. MS

Memoria de Guerra y Marina (MGM). 1860-1885. Memoria del Ministerio de Guerra y Marina de la República Argentina. Ministerio de Defensa, Buenos Aires.

Prado, M.1960 [1907].La guerra al malón. Eudeba, Buenos Aires.

Prates, L. 2009.El uso de los recursos por los cazadoresrecolectores posthispánicos de Patagonia continental y su importancia arqueológica. Relaciones de la Sociedad Argentina de Antropología 34: 201-229.

Racedo, E. 1940[1879]. La conquista del desierto. Editorial Plus Ultra, Buenos Aires.

Reigadas, M.C.1992. La punta del ovillo: determinación de domesticación y pastoreo a partir del análisis microscópico de fibras y folículos pilosos de camélidos. Arqueología, 2, pp. 9- 52.

Reigadas, M.C.2014. Explotación y aprovechamiento de la fauna en el noroeste argentino: ¿qué informan las fibras y pieles arqueológicas? Etnobiología 12(2): 64-80.

Servicio Histórico del Ejército (SHE) 1860-1885. Colección frontera con el indio. Buenos Aires.

Seymour, R.2013 [1947]. Un poblador de Las Pampas. Vida de un estanciero de la frontera sudeste de Córdoba entre los años 1865 y 1868 . Editorial El Elefante Blanco, Buenos Aires.

Tamagnini, M. y Zavala, G.2004. Las tribus ranqueles frente a los tratados de paz de la segunda mitad del siglo XIX: de las alianzas al conflicto. XV Congreso Nacional de Arqueología Argentina. Facultades de Ciencias Humanas y Ciencias Exactas, Físico-Químicas y Naturales: Universidad Nacional de Río Cuarto.

Tapia, A.2008. Arqueología histórica de los cacicazgos ranqueles. Tesis Doctoral, Facultad de Filosofía y Letras, Universidad de Buenos Aires.

Udaondo, E.1922. Uniformes militares usados en la Argentina 
J. Doval y A. Tapia | Revista del Museo de Antropología 12 (1): 23-34 | 2019

DOI: http://dx.doi.org/10.31048/1852.4826.v12.n1.20630

desde el siglo XVI hasta nuestros días. Establecimiento Gráfico Pegoraro Hnos, Buenos Aires.

Ximenez de Sandoval, C. y Madera y Vivero A. 1853.Memorias sobre la Argelia. Imprenta y estereopatí de M. Rivadeneyra, Madrid.

Yacobaccio, H., Madero, C. M. y Malmierca, M. P. 1998. Etnoarqueología de pastores surandinos. Docupress, Buenos Aires.

Yacobaccio, H., Killian, L. y Vila, B. 2003. Explotación de las vicuñas durante el periodo colonial (1535-1810). Trabajo presentado al III Taller Internacional "Manejo de Camélidos Sudamericanos: Perspectivas Arqueológicas, Antropológicas, Históricas y Biológicas. Tilcara. 